Revista Meridiano. No. 33. Universidad de Nariño, UNED. San Juan de Pasto (ISSN: 0121-0038). (pp. 257-263). 1996.

APA citation style: Benavides B., Jorge E. (1996). La Educación y la Tecnología Informática. Revista Meridiano. Universidad de Nariño, San Juan de Pasto. (33), 257-263.

\title{
La Educación y la Tecnología Informática
}

\author{
Jorge E. Benavides B. (joelbebu@gmail.com) \\ Departamento de Lingüística e Idiomas \\ Universidad de Nariño, San Juan de Pasto, Colombia
}

\begin{abstract}
Este artículo presenta una reflexión sobre la introducción de la nueva tecnología informática en educación, es decir, la inminente integración de la tecnología informática como herramienta al alcance de docentes y estudiantes en los diferentes niveles educativos. Sin embargo, este proceso, si se desea que pueda afectar positivamente la educación no se podría lograr sin la adecuada inversión tanto en tecnología como en capacitación y pedagogía. De lo contrario solo dejará altos niveles de frustración y subutilización de la tecnología principalmente en los docentes.
\end{abstract}

\section{El analfabetismo informático}

Durante las últimas dos décadas los computadores se han introducido en los diferentes sistemas educativos del mundo entero. Sin embargo, no hemos alcanzado la etapa en la que pudiéramos aplicar los computadores en educación en forma segura en nuestros salones de clase, con nuestros estudiantes para propósitos de aprendizaje. No obstante algunos profesores han hecho esfuerzos para convencer al personal administrativo, padres de familia, y a otros colegas acerca de las ventajas del uso de la informática educativa y el computador en el campo de la educación.

Tiene Ud. alguna idea de qué quise decir en las tres últimas líneas del párrafo anterior? Si la respuesta es negativa, pues es muestra de que necesitamos alfabetizarmos informáticamente. Tiene alguna idea de lo que es HARDWARE, SOFTWARE, BIT, BYTE, CHIP, MULTIMEDIA, INTERNET, utilizados de la misma forma en Inglés como en Español? Pues bien, esta puede ser otra pequeñísima muestra de cuánto ignoramos acerca de la introducción de los computadores en educación.

La primer tarea que los profesores debemos emprender es conocer y enterarnos acerca del para qué son buenos o pueden servirnos los computadores en el campo educativo; sobre sus capacidades y limitaciones como medios para entrever una posible ventana abierta hacia su aplicación en educación sin tener que traicionar los objetivos y las metas educativas. Solo así, 
Revista Meridiano. No. 33. Universidad de Nariño, UNED. San Juan de Pasto (ISSN: 0121-0038). (pp. 257-263). 1996.

nosotros como profesores podríamos iniciar el proceso de alfabetización informática aplicado a la educación, sin mención de la puramente informática como tal. De esta manera podríamos acercarnos a comprender más acerca del uso de esta nueva tecnología y contribuiríamos a que el uso del computador salga de las tareas mecanográficas ya mundanas como procesamiento de texto, el correo electrónico, hojas de cálculo y sobretodo del exagerado e indiscriminado uso de los juegos de computador por parte de nuestros estudiantes.

El conocimiento de esta nueva tecnología es pues un aspecto crítico en el adiestramiento y capacitación del profesorado moderno. Si los profesores no pueden utilizar la tecnología a causa de su desconocimiento inducido tal vez por el temor a la tecnología, por ejemplo, muy posiblemente nunca lo harán. Muchas escuelas y sobre todo colegios en la ciudad de Pasto e Ipiales en Colombia, para no ir más lejos, están haciendo lo que se hizo durante los años ochenta, en otros países, es decir, están comprando tecnología sin considerar la capacitación necesaria y su relación pedagógica, teniendo como resultado altos niveles de frustración unido a una creciente cantidad de equipos subutilizados.

El computador puede llegar a ser una herramienta de aprendizaje muy poderosa en educación pero para el laico en estos temas y más precisamente para el profesor con alto grado de TECNOFOBIA (entiéndase temor a la tecnología y al cambio tecnológico) - y que no son pocos- manejar y dominar esta nueva herramienta requiere de bastante entrenamiento. Una forma de lograrlo es tener al menos un profesor entendido (o 'experto') en esta área en cada institución educativa para que comparta y multiplique el conocimiento sobre el campo en cuestión. De esta forma el entrenamiento y capacitación con los profesores completaría su ciclo con los estudiantes. Esto es de fundamental importancia: los docentes y el personal administrativo tendrían que ser los primeros en conocer sobre la aplicación del computador en educación para manejarlo y capacitar consecuentemente a los estudiantes y por qué no a los mismos padres de familia. En resumidas cuentas la capacitación de la comunidad educativa en su totalidad tendría que comenzar con el profesor.

No es raro, y en realidad ya parece estar sucediendo, que profesores desde niveles de preescolar hasta bachillerato y universidad se encuentran cara a cara con el hecho de que sus estudiantes conocen más sobre computadores (manejo y aplicación) que ellos mismos. Esto puede ser algo desconcertante pues el creciente conocimiento global de nuestra época inclusive puede ser un factor desestabilizador para el profesor, sobre todo para aquellos que todavía consideran que ellos son la mayor autoridad en el salón de clase en cuanto a conocimiento. Muy pocos profesores, de acuerdo a mi experiencia, han sido lo suficientemente honestos para declarar y confesar que una buena parte de su conocimiento 
Revista Meridiano. No. 33. Universidad de Nariño, UNED. San Juan de Pasto (ISSN: 0121-0038). (pp. 257-263). 1996.

sobre computadores proviene de sus estudiantes. Esto por el hecho de que una creciente cantidad de estudiantes ya traen ese conocimiento desde sus hogares a la escuela.

En resumen, nosotros como profesores tenemos que pedir que se haga inversión en nuestras instituciones educativas para la consecución de equipos sin olvidar la capacitación o entrenamiento necesarios para de esta manera tener acceso al conocimiento de la nueva tecnología en educación. Este es uno de los derechos establecidos en nuestra constitución (...el derecho a la información y a la capacitación [para una mejor educación] y consecuentemente un mayor acceso a la información, según sean nuestros intereses). Esto solo puede hacerse efectivo, como lo manifiesto anteriormente, con inversión en tecnologías y al mismo tiempo con capacitación adecuada. Nuestro sistema educativo debe proveernos de estas herramientas si se desea promover el algún grado el cambio y la innovación en educación.

\section{Cuestionamientos al papel del computador en educación}

De lo único que inicialmente podemos estar seguros en cuanto al papel del computador en educación es que esta área está llena de interrogantes como cualquier campo que está por implementarse. Podría ocurrir que el computador y su tecnología pueda cambiar radicalmente la práctica educativa en cuanto a las bases fundamentales nuestro quehacer docente, es decir, el QUÉ, el CÓMO y el POR QUÉ enseñamos. Como en toda área, hay quienes afirman que lo anterior es inevitable así como los que afirman que hay que tener prudencia y estar vigilantes sobre los peligros de la introducción del computador en educación. Otros más moderados piensan que si hay algún tipo de incidencia del computador en educación esta no será tan significativa. En este caso el efecto podría ser más indirecto que central inclusive comparado con el caso del impacto de la televisión. Con este último punto de vista los profesores pueden no tener interés en utilizar el computador o solo hacerlo en situaciones muy limitadas.

Una posición que se ubicaría en medio de las dos anteriores sería la de estar abiertos a las posibilidades concretas del papel de computador y al mismo tiempo ser críticos del mismo. Esto puede ser de vital importancia para la comprensión y la práctica de la educación y los profesores deben integrarse al proceso de toma de decisiones sobre todo cuando estas si no se toman por parte de los interesados las van a tomar otros actores que posiblemente no estén tan involucrados en la práctica educativa.

Se podrían citar varias razones para explicar la gran divergencia de opiniones sobre la incidencia de la tecnología de la información en educación. Por un lado podría explicarse que la historia de la innovación tecnológica no ha producido cambios radicales en educación. Sin 
Revista Meridiano. No. 33. Universidad de Nariño, UNED. San Juan de Pasto (ISSN: 0121-0038). (pp. 257-263). 1996.

embargo, no por esta razón podríamos adelantarnos a determinar a qué nivel y en qué proporción se afectaría la práctica educativa por efecto del computador.

Una razón importante de considerar a nivel moderado es acerca del alto nivel de ansiedad que se genera debido a los cambios rápidos e imperceptibles y a la necesidad de pensar acerca del cómo podrían encajar estos cambios en los sistemas existentes con la consecuente obsesión de pensar solamente en la tecnología en sí, más no en las implicaciones de su uso en educación. Este entusiasmo muchas veces desmedido puede ser perjudicial al evaluar una aplicación y encontrar que el aporte no justifica la inversión, en dinero, tiempo y esfuerzo. Esta última parece ser la línea de pensamiento de muchos educacionalistas críticos pero que al mismo tiempo están abiertos a las posibilidades de cambio promovidos por el computador en educación. La idea predominante es que a pesar de la inversión considerable de tiempo, energía, recursos y entusiasmo durante los últimos diez años no parece haber evidencia substancial disponible para defender la idea de que la educación está cambiando en forma dramática debido al uso de la tecnología de la información.

Muchos de los defensores del papel del computador explican que debido a que estamos en una etapa inicial de introducción del uso de la nueva tecnología no puede haber un cambio significativo. La comparativamente pequeña proporción de profesores e instituciones que utilizan el computador en la práctica educativa no daría para que un efecto palpable se evidencie, es decir, es todavía demasiado temprano para que exista evidencia empírica significativa.

Lo que en realidad preocupa a gran cantidad de profesores y profesionales de la educación es, como lo mencionábamos anteriormente, es lo que tiene que ver con la capacitación del profesorado en este campo. En el momento parece difícil diseñar y desarrollar programas o aún cursos para profesores, diferentes a los de práctica con el manejo y operación del computador, por ejemplo, cómo encender el equipo, cómo poner a funcionar la impresora, cómo grabar copiar e imprimir archivos, o ejecutar programas. Todo esto con el contenido de manejo del software o programas de aplicación como procesadores de texto, bases de datos, hojas de cálculo, graficadores, simuladores, etc.

En realidad no hay que desconocer que esto es importante como un inicio en el proceso pero quizá lo más importante sea relacionar el uso del computador con el campo educativo, es decir, que no es suficiente aprender a manejar el computador sino observar su uso y aplicación en nuestro entorno educativo: tener una aproximación de indagación a este proceso como objeto de investigación. Por esta razón los profesores tenemos que aprender 
Revista Meridiano. No. 33. Universidad de Nariño, UNED. San Juan de Pasto (ISSN: 0121-0038). (pp. 257-263). 1996.

sobre lo que sucede en este contexto, tanto los defensores incondicionales de la tecnología como los escépticos o críticos.

Podríamos citar un ejemplo en el que alguien cree que el tema fundamental es el propósito de la educación y en especial su papel en el desarrollo de una mentalidad libre y crítica. Esta persona a quien la podríamos llamar un educacionalista crítico puede ver o entrever peligros en el poder que la nueva tecnología puede proporcionar a los gobiernos, por ejemplo, y a aquellos que desean incrementar la autoridad de los mismos sobre cosas tales como el juicio y la libertad individuales. Esta persona puede reclamar que se haga claridad y se distinga entre conceptos como educación, instrucción, capacitación, explotación, mercantilización, etc. A este respecto podemos observar una opinión prudente del Ministerio de Educación (1994),

... el computador ofrece potencial para el mejoramiento humano, y al mismo tiempo aparece con grandes peligros para el ser humano; en el sentido que ni su potencial pueda en realidad materializarse, ni que sus peligros puedan evitarse, sin hacer cuestionamientos críticos, cuidadosos y de alcance. (15)

\section{Aspectos de discusión de la nueva tecnología en educación}

A pesar de que algunas de las preocupaciones que se generan a raíz de la nueva tecnología surgen de la tecnología en general, o de sus productos, existen otros aspectos de tipo educativo que es necesario traer a cuento a causa de la aparición de la misma. Las implicaciones de la nueva tecnología nos deben interpelar para ponernos a pensar qué es necesario reajustar en nuestra concepción de nuestro papel y de los estudiantes en este nuevo contexto en el que estamos introduciendo además de una nueva tecnología nuevas perspectivas o nuevo entendimiento de la educación. Entre estos aspectos se pueden discutir la noción completa de currículo y de las complejas y debatidas ideas sobre la enseñanza y el aprendizaje.

Por ejemplo, al currículo lo podríamos interpretar como la suma total de experiencias disponibles para los estudiantes en la escuela y para empezar se podría considerar aspectos tales como el poder, responsabilidad y plausibilidad con respecto al impacto de la tecnología en educación. Existe un peligro consistente en que la velocidad y aparente inevitabilidad de la invasión tecnológica persuada a los profesores que ellos ya no tienen que ver en la toma de decisiones acerca del futuro de la educación o que ellos no tienen voz ni voto sobre lo que suceda en las escuelas o universidades. Sin embargo puede argumentarse con considerable fuerza el hecho de que los profesores deben continuar asumiendo su papel de dirigir tanto el paso como la dirección de la 'revolución educativa' (si se da alguna) y esto significa 
Revista Meridiano. No. 33. Universidad de Nariño, UNED. San Juan de Pasto (ISSN: 0121-0038). (pp. 257-263). 1996.

permanecer abiertos a la innovación y al mismo tiempo constructivamente críticos de la misma (Benavides, 1996).

Esto es particularmente cierto cuando se considera en nuestra época que 'la tecnología es la fuerza que mueve', ya que puede esperarse que los innovadores que se encuentran tecnológicamente acondicionados se inclinarían a mirar la educación con un alcance o foco histórico muy reducido. Los profesores, son de alguna manera los guardianes del foco más extenso, recordando que la educación tiene unas metas primordialmente humanísticas y sociales las cuales han emergido paulatinamente en un período de tiempo considerable y que estas se han construido sobre la base de la experiencia de los a veces escondidos pero cruciales constituyentes de la sociedad civilizada. Estos tienen que defenderse a veces contra las nuevas ideologías que quisieran volcar la educación en un instrumento para el logro de metas políticas o económicas particulares y temporales. Es necesario, pues que estemos vigilantes y provistos de un entusiasmo bastante moderado para ver, si la incursión del computador en educación ha impregnado velocidad a un movimiento existente que ve en la educación un proceso de re-entrenamiento en habilidades técnicas y en funcionalismo laboral.

Otro aspecto a tratar es el del énfasis en aprendizaje continuado, característica humana de por si abstracta y todavía no muy bien conocida que necesita ser tratada cuando se toca el estudio de la educación. Lo que se puede decir con certeza es que todavía no es completamente claro su significado, y no sin razón, por tratarse de un aspecto tan complejo e intrincado. Por ejemplo, en el contexto de la nueva tecnología informática en educación el peligro de la simplificación de lo que significa 'aprendizaje' se puede reconocer rápidamente tanto en el campo de la comprensión de esta característica como en el de su medición.

Papert (1980) podría considerarse un ejemplo de innovación y aplicación del potencial del computador en educación con su diseño e introducción del lenguaje y programa LOGO en la escuela norteamericana, el cual trabaja en varios niveles con enunciados epistemológicos, de 'aprendizaje' y dentro de un contexto completamente educativo. Esto es más significativo si se considera que las raíces de su trabajo se encuentran en relación al trabajo d Piaget y en el campo de la inteligencia artificial. Por ejemplo, uno de sus enunciados hipotéticos más importantes es el de que LOGO puede hacer que los estudiantes (niños en este caso) concreticen operaciones formales mucho antes de lo sugerido por Piaget y además con el ingrediente de que se hace en forma entretenida.

Sin embargo los contradictores de Papert insinúan que el peligro del uso de LOGO radica en que los niños pueden desarrollar el hábito de pensar en una forma mecánica. Sin embargo, 
Revista Meridiano. No. 33. Universidad de Nariño, UNED. San Juan de Pasto (ISSN: 0121-0038). (pp. 257-263). 1996.

según el mismo Papert, esto no podría ser desventaja si los estudiantes deciden y optan hacerlo de esta forma,

... he inventado formas para aprovechar educativamente las oportunidades de dominar el arte de pensar deliberadamente como un computador [...] Hay situaciones donde este estilo de pensar es apropiado y útil. Algunas dificultades de los niños al aprender aspectos formales como la gramática o las matemáticas derivan de su inhabilidad de entender el punto de tal estilo. [...] Al deliberadamente aprender a imitar el pensamiento mecánico el estudiante estaría en capacidad de determinar lo que es pensamiento mecánico de lo que no lo es". (p. 27)

Lo anterior se convierte en un argumento que pocas veces consideramos los profesores si nuestra posición está siempre a la defensiva. Estar abiertos al cambio, pero al mismo tiempo, decididamente críticos sobre nuestro papel en la toma de decisiones en educación podría ser una posición más que prudente sobre lo nuevo posible y lo viejo que funciona para aproximarse al cambio con criterio.

Finalmente, y con base en lo expresado anteriormente, es necesario que como profesores y profesionales de la educación estemos alertas a los posibles cambios que se puedan dar con la introducción de la nueva tecnología de la educación en la forma del uso y aplicación del computador en nuestro campo. Al mismo tiempo debemos concientizarnos que somos los primeros y los más indicados en solicitar acceso a esta tecnología para decidir y realizar la más prudente y apropiada toma de decisiones sobre el impacto de la misma en educación. De esta manera, y en forma abierta pero al mismo tiempo crítica nos convertiremos en una especie de filtro pensante, que sin traicionar las metas educativas generales, decidamos qué es lo mejor, más útil y aplicable de la nueva tecnología de la información en educación.

\section{Referencias}

Benavides B., Jorge E. (1996). Hacia una política para el uso del computador en Educación. Revista Meridiano. Universidad de Nariño. (32), 250-258.

Papert, Seymour. (1981). Mindstorms: Children, computers and powerful ideas. Brighton: Harvester Press,

MINISTERIO DE EDUCACIÓN. (1994). La Tecnología en Educación Superior. Departamento de Educación y Ciencias. Educación Superior. Bogotá: M.E.N. 